\title{
La universidad privada en la construcción de subjetividades juveniles emprendedoras
}

Private universities in the shaping of entrepreneurial youth subjectivities

\section{Leonor Lamas Zoeger}

Instituto de Estudios Peruanos / Pontificia Universidad Católica del Perú leonor.lamas@pucp.pe, leonor.lamas@iep.org.pe 


\title{
Resumen
}

Este artículo es un recuento etnográfico de los cursos y actividades de formación personal a los que tuve la oportunidad de asistir como alumna matriculada en una de las universidades privadas de segunda generación más populares del país. Este tipo de cursos ocupa hoy una parte importante de la currícula en este tipo de instituciones, y sirven de base para famliarización de los alumnos en una filosofía del éxito y el emprendimiento. Además de describir el contenido de estar materias, este trabajo se interesa por el análisis crítico de sus contenidos a partir de la teoría de la gubernamentalidad de Michel Foucault. Se analizan la relación de este discurso con la emergencia de un sujeto emprendedor, y las implicancias que tiene en la formación de ciudadanía.

Palabras clave: universidad privada de segunda generación, emprendedurismo, sujeto, ciudadanía, etnografía, gubernamentalidad

\begin{abstract}
This article is an ethnographic account of my experience as an enrolled student in one of the most popular private universities in Perú. I take special interest in describing the courses of personal education that occupy an important part of the curriculum in this institution. Based on Foucault's theory of governmentality, I aim to analyze critically the content of these courses, the way they relate with the emergence of entrepreneurial subjects and its implications for citizenship formation.
\end{abstract}

Keywords: private university, entrepreneurship, citizenship, ethnography, subject, governmentality 


\section{Universidad-empresa en la construcción de subjetividades juveniles emprendedoras}

\section{Introducción}

Los chicos llegaron con terno y corbata; y las chicas, con sastre y tacos en los $34^{\circ} \mathrm{C}$ de un mediodía de verano chiclayano. Aunque me esfuerzo por demostrar mi incomodidad entre mis nuevas compañeras de clase, mis quejas no tienen eco, pues ni el calor ni la vestimenta parecen cosa nueva para ellas, acostumbradas a ir en pantalón largo a la universidad, acordes con la solemnidad que la educación superior aún inspira en las familias chiclayanas.

Sentados ya en sus carpetas, todos los alumnos miran sus manuscritos y cierran los ojos mientras memorizan la última línea leída. A mi alrededor, mis compañeros se preguntan ansiosos los temas de sus discursos y si los han memorizado ya. Llegado el momento, la profesora comienza a llamar a los alumnos de acuerdo con el orden de la lista. Ellos salen al frente y desarrollan en tres minutos el tema de elección libre que han seleccionado para presentar.

Los alumnos controlan en diferente grado sus movimientos en el escenario, la dicción y el énfasis de sus palabras. Algunos de ellos demuestran un excelente manejo corporal, y emiten sus discursos con fluidez y naturalidad. Existen, sin embargo, casos de chicos que no manejan ninguna de las aptitudes: hablan sin despegar los ojos de sus manuscritos, en voz muy baja, tartamudeando o, incluso, con ataques de nervios que no les permiten continuar la presentación. Ante estas situaciones, la profesora interviene calmándolos, diciéndoles que se relajen, olviden al público y el discurso y digan con sus propias palabras aquello que se les viene a la mente. Después de todo, indica, por el simple hecho de haberse atrevido a «salir al frente» y combatir su miedo de hablar en público, la nota era automáticamente aprobatoria. El discurso en sí es de importancia secundaria en un curso basado en la formación de una actitud hacia el escenario y la vida

Los párrafos presentados son un fragmento de las notas de campo recogidas durante mi investigación de tesis en Antropología. Dicha investigación implicó matricularme como alumna en una de las universidades privadas de segunda generación ${ }^{1}$ más conocidas y de mayor alumnado en la ciudad de Chiclayo. Esto fue realizado con el objetivo de identificar las formas en las que el discurso del emprendedurismo -hoy, lugar común de las agencias de publicidad, y

1. Eloy Seclén (2013) agrupa bajo esta categoría a las universidades privadas creadas en el período posterior al decreto DL88. Lavado, Martínez y Yamada (2014) utilizan este mismo hito para distinguir a las universidades privadas predesregulación de las postdesregulación. 
centros de educación básica y superior- se convierte en práctica pedagógica, y moldea nuevas subjetividades y compromisos ciudadanos entre los estudiantes.

Las notas corresponden específicamente a la sesión final de oratoria, uno de los cursos que llevé durante mi primer semestre. Este es un curso que, según dicta el syllabus, busca cultivar en los estudiantes la confianza que les permita hablar en público con soltura. A ello se debe el fuerte contenido motivacional presente en muchas de las sesiones, y el énfasis que la profesora ponía en la actitud, por encima de la argumentación, en la examinación del discurso final de los estudiantes.

Junto a Oratoria, esta universidad congrega en los primeros semestres varios otros cursos de formación personal, como Tutoría, Introducción a la Vida Universitaria, y Desarrollo Personal, encaminados a fortalecer en el estudiante las aptitudes de liderazgo y autoestima que se consideran necesarias en el profesional del siglo XXI. Asimismo, la universidad organiza una gran cantidad de actividades recreativas, como ceremonias de colación, ferias vocacionales, conciertos y gymkanas. En ellas, se exige la participación de estudiantes y sus familias, y la universidad despliega estrategias de marketing para generar lealtad entre su público objetivo.

Se trata, de este modo, de un cargado calendario de actividades que no están directamente relacionadas con la formación académica de los alumnos -aquella de la que este tipo de instituciones suele adolecer-, sino orientado a la siembra de valores y aptitudes comúnmente vinculadas con el emprendedurismo, filosofía encaminada al éxito y que celebra al individuo como gestor de su propio futuro. Su introducción coincide con cambios sustanciales en la educación superior, vinculados con el proceso de desregulación del mercado educativo y a transformaciones importantes a escala económica y social. Entre estas últimas, se encuentran la emergencia de una clase media cada vez más numerosa, que ha superado la pobreza, y confía en sus atributos personales para alcanzar el progreso negado a sus antepasados ${ }^{2}$.

Como se adelantó anteriormente, este artículo tiene como objetivo identificar, desde una mirada etnográfica, la forma que toman estos cursos y actividades, así como las circunstancias que permitieron el posicionamiento del emprendedurismo como modelo de la formación personal universitaria. A lo largo de estas páginas, realizo un recuento de los contenidos y metodologías

2. En un reciente estudio elaborado por Huber y Lamas (2015), se identifican al emprendimiento y el individualismo como valores clave en la manera en la que los profesionales de la administración interpretan sus precarias e inestables trayectorias laborales. El optimismo, esfuerzo y fe en uno mismo son consideradas herramientas clave para lograr la movilidad social, por encima del reclamo político o la acción colectiva. Una situación similar es observada por Uccelli, García y Montero (2012) en estudios de caso realizados entre jóvenes de Lima Metropolitana. El emprendedurismo parece así haberse convertido en un rasgo fundamental de lo que se ha denominado las «nuevas clases medias». 
utilizadas en dichas materias, y de la manera en la que su importancia es argumentada por el personal docente y administrativo. Sosteniéndome en el concepto de gubernamentalidad neoliberal de Foucault (2008), analizo cómo estos cursos permiten la emergencia de un nuevo tipo de sujeto emprendedor, que se disciplina a sí mismo para funcionar como una empresa, que piensa en el mundo como un escenario y en la vida como un recurso a ser administrado de manera eficiente.

Una segunda línea directiva de esta investigación se pregunta por el rol que este aparato discursivo viene cumpliendo en la construcción de ciudadanía, es decir, en las relaciones que habilita entre los estudiantes y el proyecto nacional colectivo. Parto de la idea de que el emprendedurismo no es únicamente una estrategia individual para el desarrollo profesional, sino que está cargado de toda una serie de valoraciones con respecto al país y la responsabilidad en su desarrollo que hace falta explorar. A partir del marco institucional universitario y también basándome en las conversaciones cotidianas con los estudiantes, planteo, en este artículo, algunas reflexiones sobre las implicancias políticas directas de este discurso y sobre sus posibles paradojas: ¿es posible considerar a este nuevo sujeto público un ciudadano?

Esta investigación se basa en técnicas de recojo cualitativas, principalmente, la observación participante de las clases durante los primeros 3 meses del semestre 2013-1. Con el consentimiento informado de la institución, me fue posible matricularme en la universidad y participar de todas las actividades académicas en calidad de estudiante. Este permiso me permitió, a su vez, mantener nueve entrevistas a profundidad semiestructuradas con el personal docente y administrativo (tres profesores y cinco administrativos de alto rango), así como también con cinco estudiantes, con quienes la convivencia diaria me permitió establecer relaciones de confianza y horizontalidad. Mucha de la información útil a esta investigación fue extraída de conversaciones informales durante los recesos, reuniones de estudios y actividades de integración. A pesar de que muchas no fueron planeadas, estructuradas ni tampoco grabadas, fueron registradas de inmediato en mi cuaderno de campo. Por cuestiones de confidencialidad, el nombre de la universidad investigada se mantendrá en el anonimato y nos referiremos a ella a lo largo de este breve recuento con el nombre ficticio de «Universidad Emprendedora del Perú».

\section{La Universidad Emprendedora del Perú}

La Universidad Emprendedora del Perú (UEP) fue una de las muchas universidades que emergieron a fines de los años 90 a raíz del Decreto Legislativo DL882, emitido por el gobierno de Alberto Fujimori. Esta nueva normativa convertía a la educación en espacio abierto a la inversión privada con fines de lucro; y a las universidades y escuelas, en atractivas fuentes de ingresos económicos para los inversionistas. Gracias a esta desregulación, no hicieron falta más que algunos años para que el número de instituciones superiores 
universitarias aumentara explosivamente. Según cifras del último censo universitario, el número de universidades privadas y públicas ascendió a 131 en el año 2010, lo cual representa cerca del doble de la cantidad de instituciones de este tipo en comparación con las que existían en 1996 (Instituto Nacional de Estadística e Informática [INEI], 2011).

A casi dos décadas de su fundación, la Universidad Emprendedora del Perú es una de las cinco universidades privadas con mayor número de estudiantes en todo el país. Este éxito no se debe necesariamente a su buena calidad educativa. Después de todo, la UEP -y las demás universidades-empresa de su tipo- están en los últimos lugares en materia de investigación, y son continuamente cuestionadas por la poca selectividad de sus postulantes y la baja calidad de los contenidos impartidos en aulas. Su éxito está basado, más bien, en una expansión sin precedentes de la demanda educativa y en su estrategia de posicionamiento en el mercado. Las bajas mensualidades y la apertura de campus en capitales de provincia le permitieron a la UEP acoger a estudiantes migrantes de segunda generación, provenientes en su mayoría de los sectores $\mathrm{C}$ y D, desatendidos hasta entonces por las universidades públicas y privadas de primera generación, pero hambrientos por acceder a lo que era considerado la herramienta clave para la movilidad y legitimidad social: el título universitario ${ }^{3}$.

Otro factor vital en el éxito alcanzado por la UEP es la formación de una sólida identidad institucional, relacionada con los valores de emprendimiento, esfuerzo y desafío. Como bien indica Eloy Seclén (2013), estos son temas presentes en spots publicitarios, pancartas y «merchandizing» de la mayoría de universidades de segunda generación. A través de ellos, la institución educativa busca, como muchas otras hoy en día, posicionarse como una universidad de emprendedores para emprendedores, slogan que se sostiene en la historia de emprendimiento educativo de su fundador ${ }^{4}$, pero también en las historias familiares de la mayoría de sus alumnos: hijos o nietos de migrantes que llegaron a la ciudad y se abrieron paso en ella a través de empresas familiares informales.

La identidad emprendedora de la UEP, también, se hace patente en el tipo de carreras ofrecidas. Más de la mitad de ellas corresponde al rubro empresarial y las que no lo hacen tienen una clara inclinación hacia la formación de empresas propias. El trabajar para uno mismo y sin recibir las órdenes de nadie tiene en las explicaciones de la mayoría de docentes y de los alumnos una con-

3. Se trata un mito que equipara educación con progreso y que fue identificado décadas atrás para la educación secundaria (Ansión, 1993). Hoy en día, este mismo mito está involucrado en la «fiebre de diplomas» (Dore, 1976), que existe entre las clases emergentes, ansiosas por acceder al título universitario que les brinde legitimidad y status.

4. Seclén (2013) establece como una de las características claves de las universidades de segunda generación el hecho de que representen no a una colectividad, como sus equivalentes más antiguas, sino a los intereses de un promotor o fundador -en muchas, ocasiones vinculado con política-, que se vuelve bandera de la institución. 
notación moral importante. Significa ser dueño de la vida propia, del manejo del tiempo y de la toma de decisiones; es decir, permite al individuo asumir plenamente su libertad, derecho que se puede ver restringido como empleado de una organización o como dependiente de un Estado ineficiente. El imperativo de emprender se presenta, asimismo, como una estrategia clave para pasar por encima de estructuras de dominación como la clase y la raza, que le impiden a estos egresados ascender como empleados de una gran empresa. Ello queda claro al analizar algunos de los spots publicitarios más celebres de una de estas universidades-empresa: «Somos una raza distinta, que puede lograrlo todo» 5 .

Así pues, ser emprendedor implica no solo crear una empresa propia, sino también tener el coraje y empuje para decidirse a salir adelante, desafío que quedará en manos del estudiante alcanzar. El emprendedurismo se convierte, de ese modo, no solo en una estrategia económica para lograr riqueza y acceso a derechos; además, es una ética, una "antropología implícita» (Read, 2009) según la cual los estudiantes están invitados a conducir sus vidas. Es necesario, para ello, desarrollar lo que -en la literatura gerencial- se ha denominado «habilidades blandas», como el liderazgo, la proactividad y la resiliencia. Encaminados al desarrollo de esta esfera, se encuentran cursos como Desarrollo Personal y Tutoría, así como actividades extracurriculares que buscan fortalecer la creatividad, la asertividad y la autoestima de los estudiantes para que puedan afrontar con eficiencia los obstáculos que se presentan en la vida.

La existencia de estos cursos responde, también, a cambios importantes en la manera en la que se entiende la educación universitaria a nivel mundial. A raíz de los fenómenos producto de la globalización, ella se ha visto en la urgencia de pasar de modelo vertical, estructurado por objetivos, donde lo importante es la acumulación de conocimiento; a un modelo integral, por competencias, donde lo más importante no es el contenido, sino el desarrollo de aptitudes que permitan manipular, seleccionar y transformar la información recibida. Es decir, se trata de un modelo en el que el estudiante es el «agente principal de su propio aprendizaje». En este proceso, el desarrollo de lo que se ha venido a conocer «inteligencias múltiples» resulta fundamental, entre las que se incluyen el entrenamiento emocional de los estudiantes. No es coincidencia, por ello, la importancia que el consultorio psicológico tiene en la organización de actividades y en coordinar los contenidos de las clases de formación personal.

El siguiente capítulo es el resultado de mis anotaciones etnográficas de estos dos meses como estudiante emprendedora, en los cuales se observó cómo la filosofía del emprendimiento se traslada a las aulas. A riesgo de caer en un exceso de descripción, buscaré profundizar en los detalles sobre el contenido de las clases y las metodologías utilizadas por los docentes para transmitirlos.

5. Tal es un spot correspondiente a la campaña de admisión 2013 de la UCV. Un análisis discursivo de este mensaje sugiere que la educación es una herramienta que permite convertirse en una «raza distinta», ni blanca ni india, que no es objeto de discriminación. 
Para facilitar la presentación de los datos, divido este artículo en cada una de las clases de formación personal que llevé durante este período y que representan un imperativo distinto a cumplir por los futuros emprendedores.

\section{Oratoria}

«Somos valiosos, somos hermosos. Valorarse, los veo muy desanimados con ustedes mismos. Es parte de su inmadurez, pero tienen que ir adquiriendo perspectiva hacia el futuro» (profesora de Oratoria en una de las clases dedicadas al fortalecimiento de la autoestima de los alumnos).

Oratoria es uno de los cursos que componen la currícula de ciclo inicial en la UEP y es, además, una de las «actividades integradoras» que los alumnos de todas las escuelas profesionales llevan en primer y segundo ciclo. Su objetivo general, según dicta el syllabus, es familiarizar a los alumnos en el «arte de hablar en público», habilidad que es percibida como fundamental en un buen estudiante y profesional del siglo XXI.

Del objetivo general se desprenden, a su vez, dos objetivos secundarios. En primer lugar, se apunta a fortalecer a través de distintas dinámicas la confianza y el autoestima de los recién ingresantes, que les permitan afrontar su miedo escénico. En segundo lugar, el curso está enfocado en el aprendizaje de técnicas de persuasión y manejo del escenario que recuerdan a aquellas utilizadas por actores y músicos para adquirir seguridad frente al público. Es decir, el curso está enfocado en el desarrollo de habilidades psicológicas y dramáticas que permitan el control del escenario, más que en las técnicas de argumentación, que son dejadas en segundo plano. La atención está, así, en la performance del decir, más que en el contenido de aquello dicho.

De este modo, las primeras sesiones estuvieron dedicadas a la elaboración de dinámicas para desarrollar la autoestima y la seguridad de los alumnos. Las sesiones, por lo general, comenzaban por invitarlos a sentarse en círculo en el salón con la profesora al medio, quien de inmediato comenzaba con un encendido monólogo motivador, en el que se enfrentaba al alumno con una manera distinta de ver la vida y proyectarse hacia el futuro. Esta nueva filosofía era presentada como un factor fundamental no solo para adquirir confianza en el escenario, sino también como un requisito para la «felicidad» y la realización personal.

Entre los lineamientos brindados a los alumnos, estaba la adopción de una actitud asertiva hacia la vida. Una actitud asertiva corresponde a una persona optimista, que no se deja amilanar ante las críticas ni los obstáculos. La profesora invitaba continuamente a los alumnos a reconocerse como personas valiosas, sin importar su procedencia, clase social ni las dificultades que se pasaron en la vida. «Somos hermosos y completos» y "Amémonos y respetémonos» eran frases recurrentes en el monólogo de la docente, quien 
proseguía pidiéndole a cada uno de los alumnos que identificasen sus cualidades y cómo podrían ser de importancia fundamental para la vida de quienes los rodean.

Asimismo, la docente añadía: «El "camino al éxito" estará indudablemente lleno de obstáculos e inconvenientes que de ningún modo deben detenernos. Problemas como la pobreza, la vulnerabilidad y la violencia familiar, más que como barreras, deben ser interpretados como retos que es necesario cumplir y que hacen de la victoria aún más gratificante». El carácter individualizado y performativo de la manera en la que los alumnos se enfrentan a estos «retos cotidianos» es la misma que la utilizada por el orador al enfrentarse a una audiencia -a la cual debe cautivar usando ingenio, y dejando atrás las penas y malos momentos de la vida real-.

Además de motivación, el curso también está enfocado en el desarrollo de habilidades histriónicas de manejo del cuerpo y el escenario. Un buen orador debe estar consciente siempre de que su imagen y actitud corporal son fundamentales para conectar y persuadir al público. Por ello, debe, en primer lugar, controlar su aspecto personal, preocupándose por estar limpio y bien ordenado, de manera de que su imagen inspire respeto y autoridad frente al público. Por ello, en el examen -y, en general, en todas las presentaciones a lo largo de la carreras universitaria-, es obligatorio que los alumnos se presenten en terno o sastre, zapatos de vestir y correctamente peinados.

Así como su vestimenta, el "performer» debe también desarrollar control sobre sus movimientos y reflejos durante la presentación, para lo cual se recomiendan ejercicios de respiración, gritos y trabalenguas, que relajan los músculos y mejoran la dicción del orador. Asimismo, para incrementar la confianza y evitar la ansiedad, se sugieren ejercicios de autosugestión y relajación previa, así como prácticas constantes frente al espejo o una grabadora, los cuales permiten al orador estudiar y optimizar lo que serán sus movimientos en el escenario. Ello responde a que, una vez ahí, el cuerpo ya debe estar completamente disciplinado. Al mensaje lingüístico -de importancia secundaria- debe acompañar una serie gestos, miradas, movimientos coordinados que atraigan la atención de la audiencia y la persuadan a seguir escuchando. En simultáneo a la presentación del argumento, se debe, por ejemplo, mantener un caminar pausado y seguro por el escenario; se debe cuidar que este no se convierta en una acción compulsiva que demuestre inseguridad. Del mismo modo, el contacto visual directo con el público es muestra de solidez y genera empatía. En cuanto al movimiento de manos, siempre que se mantenga discreto y pertinente, este llama la atención del público y permite hacer más expresivo el mensaje que se quiere transmitir.

Así pues, como deja ver esta breve discusión del contenido de las clases de Oratoria, tanto los ejercicios de motivación como los de control del escenario están enfocados en asegurar una presentación efectiva de los discursos, que cause impacto y recordación en la audiencia. Ello se basa más en la «actitud» y las estrategias de persuasión, que en la argumentación o el contenido de la 
exposición. Se trata, por lo tanto, de una disciplina eminentemente performativa, que toma sentido únicamente en términos de eficacia, eficiencia y efectividad (Mckezie, 2001). La importancia que se le da al medio y al «hablar» por encima del contenido quedó clara en las indicaciones para el examen final de la clase; este consistía en un discurso de tema libre en el cual sería necesario demostrar las estrategias de presentación y persuasión aprendidas en clase.

En este ejercicio, mi idea fue ser, en efecto, polémica. Mi intención premeditada fue aprovechar la plataforma de «tema libre», abierta por la docente, y tocar un tema por lo general silenciado, del que no se «habla» con facilidad en las esferas públicas, como es el matrimonio igualitario. Las reacciones de la profesora y mis compañeros demostraron ser fundamentales para encontrar las paradojas de un discurso, a primera vista, democratizador y participativo.

Después mi presentación -en la que, también, me serví de las técnicas de persuasión y control del cuerpo aprendidas en clase-, la profesora se detuvo a plantear algunas aclaraciones que se relacionaban indirectamente con el contenido. Llamó la atención, por ejemplo, sobre el cuidado que debía tener un orador al tocar temas polémicos. Estos, en lugar de cautivar al público, podían más bien generar resistencias, espantar e indignar, reacciones que sin duda eran opuestas al objetivo central de un arte como la oratoria, que apunta a cautivar y embellecer. Mi discurso, en este sentido, por más que había sido preformado correctamente, habría perdido eficacia por el carácter incómodo de su contenido.

Asimismo, la profesora sugirió que un discurso como el mío era, en pocas palabras, una falta de respeto, debido a que hería susceptibilidades y «confrontaba» a la audiencia con ideas polémicas, que esta no estaba en condición de responder. Utilizar el escenario cedido como espacio para «imponer» ideas políticas era interpretado casi como un ejercicio arbitrario de poder, incluso, moralmente incorrecto, aun cuando ese mismo espacio hubiese sido utilizado ya para presentar ideas más inofensivas sin la misma censura ${ }^{6}$.

La contundente reacción de la profesora me hizo reflexionar sobre las verdaderas implicancias del imperativo de hablar comprendido en un curso como el de Oratoria, inicialmente planteado como un espacio libre y abierto a las propuestas de cualquiera, incluso como una especie de laboratorio para el ejercicio pleno de la ciudadanía. Después de todo, «hablar» y «ser escuchado» recordaba a los reclamos progresistas por participación en todas las esferas de toma de decisiones y por una ciudadanía activa, "que hace escuchar» sus reclamos. Sin embargo, frente a estos acontecimientos, ¿era posible seguir pensando en el «escenario» como un espacio democrático?

Uno de los comentarios de la profesora, que encontré bastante escondido en mis notas de campo, me ayudó a ensayar una respuesta a esta aparente paradoja:

6. Entre los temas más comunes presentados esa mañana por los alumnos, estaban el cuidado del medio ambiente, la condena del alcoholismo o la narración de anécdotas chistosas o conmovedoras. 
Ustedes tienen que empezar a hablar. Que se note su voz, que se note su presencia. La Universidad transforma. Así que, chicos, ustedes son el futuro, son más de mil, son lo que la universidad quiere dejar: gente competitiva, dinámica, gente que hable, que se produzca, que sepa pedir, que sepa hacer, que sepa solucionar. No que solo se queje, se queje, se queje ¡NO! Sino que, a partir de un problema, emerja una solución. ¿Es viable o no plantear alternativas? (profesora de Oratoria).

Como es posible detectar en las palabras de la profesora, dichas en una de las sesiones de motivación, el imperativo de hablar al que éramos convocados sí estaba restringido, en primer lugar, por las barreras de lo consumible y lo aceptable. Lo dicho debía estar siempre acorde con las preferencias de la audiencia generalizada, quien es, en último término, la jueza de la pertinencia de nuestras ideas.

Ello no quiere decir, sin embargo, que no haya espacio para la innovación en las propuestas del orador, a quien -más bien- se le incentiva a la creatividad y al pensamiento divergente. No obstante, estas propuestas debían estar en tono «no confrontacional». Es decir, si bien el sistema puede ser cuestionado, no puede plantearse como «queja permanente», sino articularse en forma de propuesta, argumentada en términos técnicos económicos de viabilidad y sostenibilidad, en otras palabras, funcionalidad ante un sistema que debe permanecer a grandes rasgos, incuestionado. Aquellos reclamos que no se puedan articular en estos términos son considerados «políticos», con toda la carga negativa que ello implica.

\section{El imperativo de la gestión personal}

Además de un buen orador, un emprendedor debe ser un excelente gestor y planificador de su propia vida. Este es el mensaje que se desprende de las clases de Desarrollo Personal y de los diversos talleres organizados por el Consultorio Psicológico, enfocados en la optimización del uso del tiempo, la mejora de los procesos aprendizaje y la elaboración de un «plan de vida». Este último punto, según es explicado en las clases, consiste en el planteamiento de metas realizables a corto y largo plazo, que sirvan de horizonte de motivación para el estudiante, y que le permitan organizar su tiempo y decisiones. Establecer metas supone, primero, una autoevaluación completa de las aspiraciones del individuo, que deben ser operacionalizadas en metas realizables más pequeñas. Por ejemplo, si un estudiante tiene como aspiración graduarse de la universidad, tendría primero que aprobar sus cursos de primer ciclo, lo cual -a su vezrequiere dedicar dos horas semanales de estudio a cada una de las materias. Una vez jerarquizadas y graficadas, el estudiante obtiene un «árbol de metas» -o «árbol de objetivos»-, que brinda la clave operativa para alcanzar el éxito en las metas propuestas. 
Realizado este ejercicio, el alumno es invitado a realizar un análisis FODA (fortalezas, oportunidades, debilidades y amenazas) de su situación actual. Es decir, debe identificar los recursos con los que cuenta para cumplir sus metas, las dificultades que se podrían presentar en su camino a cumplirlas y los posibles riesgos implicados en este proceso. Este análisis permitiría planificar una estrategia clara y objetiva para alcanzar las metas, así como un esquema económico del costo y beneficio involucrado en cada una de estas. Ello lleva a una jerarquización aun más fina de las mismas, de acuerdo con criterios económicos, que -después- pasa por un proceso más de sofisticación según criterios de urgencia y prioridad.

La materialización última de este proceso de planificación consiste en un horario semanal con un resumen detallado del uso diario del tiempo, enfocado en el cumplimiento de las metas planteadas. La elaboración y presentación de este horario fue la tarea asignada a los alumnos como evaluación de la primera parte del semestre. Las metodologías utilizadas para planificar la vida de los alumnos y controlar el uso de su tiempo me hicieron recordar mucho a lo aprendido durante mis cursos de gestión de proyectos de desarrollo, en los cuales los mismos análisis son utilizados para cuantificar la pertinencia y establecer el plan operativo de una determinada iniciativa social. Según fue mencionado por el profesor de la clase, este mismo método es utilizado también por un sinnúmero de empresas exitosas para asegurar un funcionamiento eficiente y crecimiento. La propuesta del curso pasaba, por lo tanto, por trasladar los mismos sistemas de gestión organizativa a la planificación de la vida diaria de estos estudiantes, convirtiendo sus proyectos personales en pequeñas empresas a ser gestionadas bajo los criterios de pertinencia, operacionalidad, eficiencia y productividad.

Así pues, como se vio anteriormente, la planificación de la vida según análisis FODA implica posicionar las habilidades, aptitudes y experiencias del individuo como recursos a partir de los cuales se puede alcanzar las metas planteadas. Es decir, la vida de los jóvenes debe ser entendida como capital humano, capaz de ser invertido, transformado, optimizado para aumentar su eficacia en la obtención de un título universitario o de un trabajo bien remunerado. Ello implica una responsabilidad del individuo sobre la correcta gestión de su capital, sea cuidando su cuerpo, mejorando sus hábitos de estudio, controlando sus tiempos o desarrollando habilidades que lo hagan más competitivo en el mercado laboral. En la gestión correcta de estos capitales, se esconde la clave para alcanzar el éxito y la realización personal.

De ello se desprende que el fracaso se debe, también, a las negligencias del propio administrador y nunca a factores externos; estos, dentro de esta perspectiva, son tomados como riesgos medibles, que pueden ser evitados o minimizados con eficiencia si tan solo se movilizan los recursos adecuados. De este modo, se invisibilizan los obstáculos y la estructura: todo depende de uno mismo. Así, se refleja en los incontables mensajes publicitarios, en los cuales 138 I los obstáculos se minimizan ante la voluntad y motivación del estudiante. 


\section{Tutoría y «bienestar estudiantil»: El imperativo de participar y la identidad de marca}

Como se esfuerzan por enfatizar la mayoría de docentes a los que tuve la oportunidad de entrevistar, el sistema de tutorías es una de las principales innovaciones de la Universidad Emprendedora en materia pedagógica. Este curso nace muy temprano en la historia de la institución ante la alta proporción de alumnos que abandonaban sus estudios en los primeros ciclos, debido principalmente a dificultades económicas, pero también a problemas de adaptación a la vida universitaria, oposiciones familiares y fracasos académicos ${ }^{7}$. Frente a esta situación, que ponía en peligro la sostenibilidad de la empresa educativa, el fundador tomó la decisión de crear un sistema encaminado a realizar seguimiento pedagógico y psicológico de los alumnos durante los primeros dos años de la carrera; de este modo, podía asegurar su permanencia en la institución.

Nace así el curso de tutoría, a cargo de psicólogos profesionales a quienes se les asigna monitorear y servir de mentores para un mismo grupo de estudiantes a lo largo de cuatro ciclos académicos. Estos tutores son responsables de mantener un control permanente sobre las asistencias de los alumnos, su rendimiento académico y su estado emocional, para lo cual se comunican continuamente con los docentes, administrativos y padres de familia. Esta triangulación constante de información les permite detectar problemas que interfieren en el desenvolvimiento de los jóvenes, y buscar soluciones rápidas y coordinadas para incrementar su bienestar.

El curso de Tutoría está encaminado así a asegurar la atención integral de los estudiantes para aumentar su comodidad con la institución. Ello representa un cambio significativo en la manera en la que hasta entonces se entendía la relación estudiante-universidad. De un modelo escolástico, de lenta transformación y en el que la vida del estudiante -fuera de su desarrollo cognitivoquedaba desatendida, pasamos a otro: un modelo organizacional, en el cual la universidad se ve en la obligación de optimizar su servicio para mantener cautiva la demanda estudiantil. Estas mejoras, sin embargo, no se dan a nivel de docencia y contenidos -aspectos en los que se encuentran las verdaderas deficiencias del servicio-, sino a un nivel que -a falta de otro concepto- denominamos «cultural», en la medida que supone el desarrollo del bienestar subjetivo de los alumnos y de nuevas formas de identificación organizacional.

Esta transformación empresarial del servicio educativo privado implica, según la literatura especializada, que la universidad comience a ser entendida como proveedora y el estudiante como cliente. Mi experiencia del

7. Si bien la Universidad Emprendedora no suele expulsar a sus alumnos por desaprobar cursos, sí impone sanciones económicas para los estudiantes repitentes, que consisten en el pago de 100 soles por cada curso desaprobado. Antes de la introducción de los cursos de tutoría, la tasa de deserción llegaba al 50\% del estudiantado, según datos ofrecidos por el director académico de la UEP. Hoy esta cifra se ha reducido a la mitad. 
funcionamiento organizacional de la UEP sugiere, sin embargo, que el papel que mejor calza con el del estudiante en esta estructura es el de empleado, principalmente, por no tener el empoderamiento para influenciar en las ideas de la empresa y -como veremos más adelante- por ser uno de los recursos más importantes para su posicionamiento en el mercado.

Así, en este mismo esquema, el curso de Tutoría y la red de servicios estudiantiles que articula podrían bien ser análogos al área de Recursos Humanos de la institución, en la cual se elaboran los mecanismos para hacer de esta última no solo un espacio para la adquisición de conocimientos, sino también para el disfrute y la realización personal de los empleados/estudiantes. Estos mismos recursos son movilizados hoy en día por un sinnúmero de empresas de servicios que -bajo el paradigma de "performance management»- se esfuerzan por hacer del lugar de trabajo un espacio en el que el empleado se sienta a gusto de realizar sus funciones (Danzelot, 1991).

Los primeros semestres de la clase de Tutoría están encaminados al desarrollo de una identidad institucional entre los estudiantes, que los profesores denominan «espíritu UEP». Se trata de un proceso de «inducción» o «fidelización» semanal, que recuerda a aquellos por los que atraviesan los empleados de las empresas antes de iniciar sus labores. El desarrollo de esta identidad es uno de los criterios más importantes para la evaluación del curso, que, si bien no cuenta con creditaje, es de aprobación obligatoria para la graduación.

Además de las clases de Tutoría, la Oficina de Bienestar Estudiantil y el Consultorio Psicológico organizan regularmente actividades de recreación para los alumnos, encaminadas a integrarlos al resto de sus compañeros de clase y profesores. Entre las actividades en las que participé durante mi estancia en la UEP, se encuentran una gymkana de bienvenida a los alumnos del ciclo Alfa, el Día del Psicólogo, el cumpleaños del fundador, entre otras pequeñas dinámicas de integración y conocimiento mutuo que llevan a cabo los tutores durante sus horas de clase. Además de estas, la directora de la Oficina de Bienestar Estudiantil me comentó de la existencia de las actividades integradoras por el Día de la Juventud, el Día del Folklore y el aniversario de la universidad. 


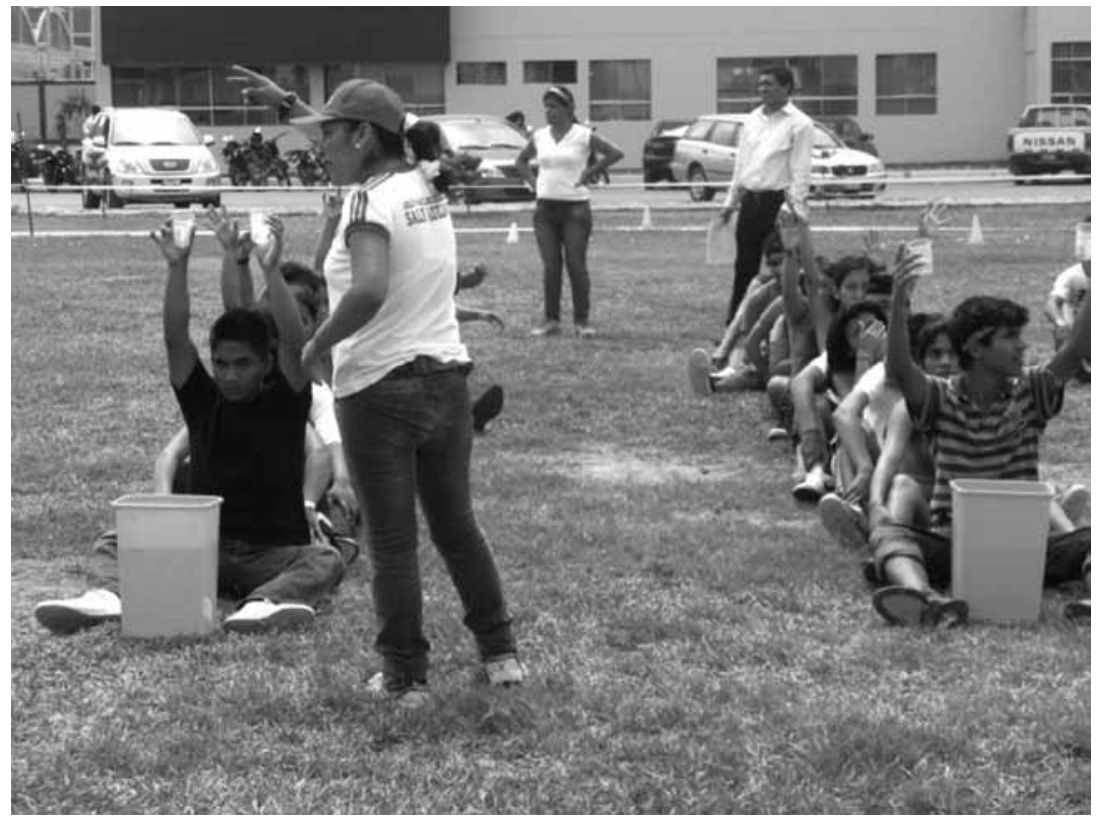

Figura I. Gymkana de integración ciclo Alfa - Competencia «Pasando el agua»

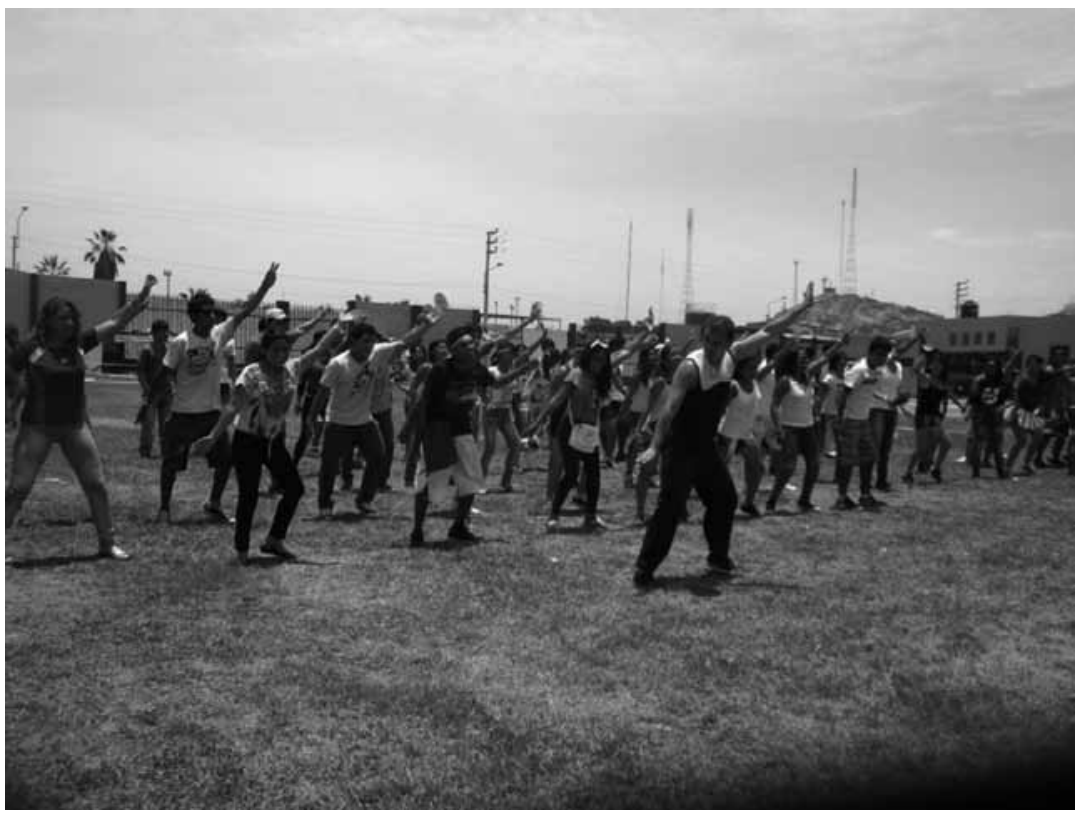

Figura II. Gymkana de integración ciclo Alfa - Clases de danza moderna 
Como alumna, participé y disfruté mucho de las actividades, pues, efectivamente, me permitieron socializar con mis compañeros por primera vez $y$ establecer los primeros contactos para mis próximas entrevistas. Sin embargo, llamó mi atención, en primer lugar, la cantidad de recursos movilizados para su montaje. En vista de que la mayoría de estas actividades congregan a por lo menos una especialidad entera de alumnos y ocupaban casi toda la jornada de clases, ellas requieren de la participación de casi todo el personal docente y psicológico de la especialidad en cuestión, quienes asumían la tarea de organizar a los alumnos (formación de equipos para las competencias, etc.), movilizarlos a las canchas y controlar su comportamiento. Ello contrastaba notoriamente con lo que ocurría en universidades como la mía, donde, si bien existían actividades integradoras para los alumnos -especialmente durante los primeros ciclos-, se trataba de iniciativas gestionadas enteramente por las organizaciones estudiantiles o los centros federados de cada facultad ${ }^{8}$.

En la UEP, en cambio, estas actividades son entera iniciativa de la institución, lo cual sugiere que estas forman parte de un objetivo expreso por formar una sólida cultura organizacional entre sus alumnos. Ello es más evidente si tomamos en cuenta que la participación en estas actividades es para los estudiantes muchas veces obligatoria y evaluada, como señala la profesora Rosa. En su participación constante y en la actitud demostrada durante las competencias, se evalúa no solo su identificación con la institución, sino el desarrollo de los valores y habilidades que caracterizan al «espíritu UEP».

Se podría aventurar la interpretación de que se trata de una nueva manera de fundar la "comunidad universitaria», sobre la base ya no del conocimiento y el debate, sino del sentimiento compartido de pertenencia a una misma institución y a los valores que ella profesa. Este nuevo sentido de colectivo se funda en la acción reiterada de este tipo de rituales corporativos.

\section{El líder, un nuevo modelo de ciudadano}

«La universidad, por lo tanto, no debe ser vista únicamente como una fábrica de formar profesionales, con una misión egoísta, con una visión mercantilista. No. La universidad tiene formar profesionales competitivos, sí, que les dé rédito económico, sí, pero sobre todo solidarios, participativos, preocupados, por hacer del espacio donde se desarrollan, un espacio digno de vivir» (director general UEP).

La Universidad Emprendedora del Perú, conforme ha ido tomando protagonismo en la esfera pública, ha ido también articulando un discurso

8. Ello, sin embargo, parece estar cambiando. En los últimos años, en la Pontificia Universidad Católica del Perú (PUCP), se ha estado realizando una bienvenida de cachimbos que -además de la usual ceremonia de bienvenida con el rector-implica ahora conciertos, juegos y actividades de todo tipo, que se encaminan a dar al recién ingresante una primera buena impresión de lo que será su estadía en la universidad. 
con respecto al papel que cumple en la sociedad civil y en la formación de futuros ciudadanos. Parte de este proceso -como denota la cita- ha supuesto diferenciarse del resto de universidades privadas de segunda generación -que son acusadas de estar únicamente enfocadas al lucro-, y posicionarse como una empresa socialmente responsable, formadora de ciudadanos sensibles y comprometidos con el desarrollo de su país.

El aprendizaje-servicio es un sistema manejado por la Oficina de Proyección Social, que forma parte -a su vez- del Vicerrectorado de Asuntos Estudiantiles (VAE). Esta oficina tiene como objetivo articular los procesos de investigación de los alumnos a través intervenciones sociales en comunidades de bajos ingresos. En el año 2013, el proyecto piloto de esta iniciativa fue llevado a cabo en la comunidad «Nuevo Porvenir», ubicada al frente de la universidad. Con ayuda de un "profesor eje», que se mantiene por los diez ciclos académicos, los estudiantes diseñan y aplican proyectos de investigación encaminados a mejorar las condiciones de vida de la comunidad, a nivel psicológico, educativo, legal o social.

Por ejemplo, en 2012, los estudiantes de Educación se dedicaron a concientizar a las familias sobre la importancia de la educación no formal, los abogados dieron consultorías a familias en proceso de titulación de terrenos y los ingenieros ambientales trabajaron con la comunidad estrategias de manejo de residuos sólidos. La diversidad de especialidades que trabajan en el mismo lugar ha asegurado la integralidad de la intervención.

Tal como ha sido planteada, la metodología se muestra beneficiosa tanto para el aprendizaje del alumno como para la comunidad de incidencia. En primer lugar, el modelo de aprendizaje-servicio permite al estudiante aplicar a la realidad los conocimientos aprendidos en el aula, haciendo de su aprendizaje mucho más significativo y acorde al enfoque de educación por competencias descrito con anterioridad. Es decir, permite desarrollar en los jóvenes mayor autonomía en el acceso al conocimiento y responsabilidad en la manera en la que este se vuelca a la realidad.

Por otro lado, la intervención, según comenta la profesora Ana, también está encaminada a fortalecer la autonomía de la población beneficiaria, a quien desde un inicio se le pide su participación e involucramiento activos en el desarrollo del proyecto. Como indica la profesora, se trata de un gran paso en relación con la manera en la que se pensaba la proyección social anteriormente: de un modelo asistencialista, en el que los beneficiarios recibían pasivamente la ayuda de terceros; a uno de empoderamiento, en el cual lo importante-también- es el desarrollo de las competencias que permitan a la población agenciar su propio desarrollo de manera sostenible. Ello, evidentemente, implica un "cambio de paradigmas» en la población, que también se ha propuesto lograr la UEP a nivel regional, mediante un proyecto que busca extender los grupos de líderes a las escuelas secundarias de todo Lambayeque.

El modelo de aprendizaje-servicio supone, además, una nueva manera de entender la participación de la universidad en la sociedad civil. Esta no es 
indiferente al contexto social en el que se desarrolla, pero tampoco guía su modelo de incidencia de acuerdo con las universidades tradicionales, en las que el compromiso social de la institución se mide a partir de la calidad del debate interno y politización. La UEP, a través de su Oficina de Proyección Social, propone -más bien- un involucramiento directo con la realidad social mediante «acciones» concretas, que puedan tener resultados medibles en el bienestar de la población objetivo. Es decir, se trata de un modelo de compromiso social guiado, también, por los imperativos tánicos de eficacia, eficiencia y efectividad, esta vez aplicables a la esfera social y política. El compromiso y la conciencia social se muestran pertinentes de acuerdo con su aplicabilidad y operacionabilidad, y usa en su desempeño criterios organizacionales que han sido evaluados anteriormente (como el árbol de objetivos, análisis FODA, etc.)

La existencia de este tipo de compromiso universitario supone la existencia de jóvenes ciudadanos más activos, y capaces de asumir proactiva y responsablemente su papel en la sociedad. Todas estas cualidades se materializan en la figura del líder, a cuya formación se avoca la UEP. Así, pues, la Oficina de Proyección Social tiene a su cargo la agrupación Líderes en Acción, único grupo de estudiantes de la UEP con fines no académicos ni recreativos. Este grupo está conformado, hasta el momento, por trece alumnos seleccionados por la profesora Ana, debido a su notorio compromiso con las actividades de la universidad y su gran sensibilidad en el trabajo con las comunidades. Ellos representan, como su nombre lo dice, el espíritu de «acción» que caracteriza a la nueva responsabilidad social universitaria y al «espíritu UEP» participativo del que nos detuvimos a hablar con anterioridad.

Estos jóvenes se reúnen semanalmente a organizar actividades de incidencia en las comunidades cercanas, hacer seguimiento a los proyectos de aprendizaje-servicio y, sobre todo, representar a la universidad en plataformas de toma de decisiones, como el sistema regional de juventudes, en cuyo diseño participaron unas semanas antes de mi llegada al campo. Asimismo, son uno de los principales contactos entre los alumnos y las autoridades universitarias, y se preocupan por hacer llegar las iniciativas y preocupaciones de sus compañeros a las oficinas administrativas. Su función es, pues, lo más cercana posible a un centro de representación estudiantil (inexistente en la universidad), aunque su influencia no se siente a nivel de política universitaria, sino que se articula en los términos de la Oficina de Bienestar Estudiantil.

Un rasgo fundamental en la figura del líder, según nos comentaron los docentes, es su participación. Un líder es una persona proactiva, que no cae en la pereza, y que se conmueve y responsabiliza por las carencias de su sociedad. No espera a que un Estado ineficiente genere el cambio social, sino que está dispuesto a «tomar cartas» en el asunto. Un líder así es la figura del emprendedor ciudadano, que no solo asume control sobre su vida de manera autónoma y eficiente, sino que busca utilizar esos mismos atributos para lograr cambios significativos su sociedad. 
Entrevistas colectivas que también formaron parte de la elaboración de mi tesis demostraron, sin embargo, que este compromiso por cambiar las condiciones de existencia de la comunidad no se articulan en clave de la política partidaria. Los estudiantes no muestran interés por sumar sus iniciativas sociales a ningún partido político. Por el contrario, condenan la ineficiencia y corrupción usualmente vinculadas a estas esferas de la toma de decisiones. Su llamado a «iniciar el cambio desde uno mismo» está relacionado así a la conciencia de un Estado en el que -como se ha mostrado por años- no se puede confiar en lo que respecta al manejo de la nación, y que requiere a gritos la eficiencia tecnocrática de un gerente, no de un presidente convencional.

El país es una gran empresa. Mira, una empresa se divide en distintas áreas. Para que las distintas áreas funcionen perfecto y la organización crezca cada día más, ¿qué es lo que necesita?: orden, responsabilidad y gente que cumpla cabalmente sus funciones. Eso se divide así, como el país: el país es una gran empresa, que está dividida por áreas -que son sus veinticuatro departamentos- $y$, para que todos sus 24 departamentos funcionen bien, todos deben estar en coordinación y constante crecimiento. Tengo un pensamiento así, pero creo que es la mejor forma de mostrar con ejemplos lo que podía hacer para desempeñar funciones y administrarlo mejor (Manuel - estudiante de Economía).

Desde esa perspectiva, para los jóvenes líderes, el Estado no debe hacer más que gerenciar; cohesionar; administrar la acción autónoma, libre y responsable de ciudadanos. Aquel que asuma el gobierno del país tiene -a la vezque asumir ese compromiso por la formación de estos nuevos ciudadanos. Ello se debe realizar a través de un nuevo tipo de educación, pero también de un ejemplo, un líder que -encarnando los valores que caracterizan esta figura- invite a los ciudadanos a seguir su misma senda.

\section{Conclusiones y discusiones}

Este artículo es un breve resumen de una etnografía realizada en una de las universidades privadas de segunda generación más grandes y con mayor relevancia mediática en el país. Las limitaciones de espacio no nos han permitido ahondar detalles ni interpretaciones teóricas; sin embargo, las descripciones seleccionadas son claras y, en muchos casos, «hablan por sí solas». En cualquier caso, se trata de un primer paso en la aproximación empírica y etnográfica a instituciones cuya importancia en la literatura especializada no es proporcional con el peso que han ido cobrando para el país en los últimos diez años. Se trata, también, de una crítica al discurso del emprendimiento desde el mundo empírico, tarea que se muestra urgente ante la fuerza ideológica que este ha ido tomando en los últimos años en la definición de la nación y la ciudadanía (Cánepa, 2012, 2013). 
Hemos demostrado hasta este punto que la UEP ha capitalizado su extracción popular para posicionarse como una universidad emprendedora. Este emprendimiento no es solo una estrategia económica, sino también una filosofía de vida, que enaltece la autonomía y responsabilidad del individuo en la construcción de su propio futuro. En materia pedagógica, este discurso supone una toma de protagonismo del individuo en los procesos de aprendizaje, y una mayor relevancia de los elementos emocionales y culturales en la construcción de conocimiento. En la UEP, ello se ha visto plasmado en una currícula que dedica una buena parte de sus horas lectivas al fortalecimiento de habilidades como la oratoria, la gestión de la vida y el desarrollo de una identidad organizacional.

La emergencia de estas tecnologías del manejo de la subjetividad responde, para Michel Foucault (2008), a un cambio en la forma de ejercer el poder durante el liberalismo. Lejos de ser difundido desde un centro político y administrativo en forma de reglamentos y castigos -como sucedía en la Edad Media-, el poder, desde el siglo XVIII, se caracteriza por la descentralización de la capacidad de control del Estado a los individuos, quienes a partir de entonces juegan un rol más activo en el gobierno de su propio cuerpo y pensamientos. A esta nueva economía del poder, basada en la regulación «desde dentro» de la población, Foucault la denomina «gubernamentalidad» (Foucault, 1999).

Llegado el neoliberalismo, los sujetos comienzan a ser gobernados como agentes del mercado competitivos, «incentivados a cultivarse a sí mismos como individuos autónomos y egoístas, y pensar en sus recursos y aptitudes como capital humano para inversión y ganancia» (Hamman, 2009, p. 50). Desde esta perspectiva, el sujeto del neoliberalismo piensa en sí mismo como una empresa y en la vida como un mercado competitivo. Sus decisiones, vinculadas con el trabajo, la educación, el cuerpo o el tiempo libre, son tomadas como inversiones de capital encaminadas a maximizar el dinero o satisfacción. Foucault bautiza este nuevo sujeto neoliberal como el «homo economicus» o el emprendedor de sí mismo. Resulta difícil no encontrar las analogías con los discursos institucionales de la UEP analizados en este trabajo.

La promesa de libertad asumida por el emprendedor, sin embargo, no es irrestricta. A la individualización ejercida por las instituciones de control acompañan mecanismos de "conducción de la conducta», que establecen límites deseables para el ejercicio de la libertad individual, reproduciendo categorizaciones, jerarquías y regímenes de poder. El caso de la Universidad Emprendedora demuestra claramente, en cada una de sus materias, cómo el imperativo por emprender viene acompañado de una serie de exigencias sobre el manejo de la vida propia y de una forma particular de entender la política que son también excluyentes y restrictivas.

Con ello, no quiero decir que todo intento de desarrollo del emprendimiento, de las habilidades de hablar en público y de una identidad colectiva reproduce el sistema neoliberal, y merece ser objeto de crítica. Es necesario dejar 146 I eso claro a los legítimos defensores de este tipo de estrategias para mejorar la 
autoestima y el sentido de pertenencia de comunidades afectadas por la pobreza y la violencia, como es el caso de muchos de los estudiantes de la UEP. El problema surge cuando este discurso se vuelve normativo, y oculta tras sus aparentes beneficios mecanismos de exclusión y de censura. En cierta medida, fue eso lo que presencié en la UEP, institución que mediante sus cursos y actividades contribuye a la formación de un sujeto con las siguientes características:

- Un sujeto asertivo y participativo, pero que performa en un escenario parametrado por lo consumible y aceptable: La pretendida democratización del derecho a hablar ofrecida por un curso como el de Oratoria se ve limitada ante la restricción a la que se enfrentan las voces divergentes, aquellas que no pueden ser transmitidas con la misma efectividad que aquellas que se inscriben en consensos sociales. De este modo, es visible la manera en la que los argumentos de eficiencia comunicativa, aparentemente técnicos e imparciales, sirven en la práctica para silenciar prácticas y discursos incómodos.

- Un sujeto que planifica y gestiona de manera responsable, y en términos de inversión y ganancia todas las esferas de su vida, incluso aquellas que antes no eran evaluadas como capital: El éxito y la oportunidad de salir adelante quedan condicionadas exclusivamente al esfuerzo propio y a la capacidad de gestionar correctamente los recursos. En este sentido, se invisibilizan las barreras aún existentes entre estos emprendedores y la tan ansiada movilidad social, entre ellas, la baja calidad de la educación superior que reciben en la universidad. Esta aproximación a la vida en términos de capital individualiza problemas como la desigualdad y la pobreza, y legitima el orden de la sociedad, es decir, del régimen neoliberal que se ha instalado en el país.

- Una colectividad que se conforma en espacios rituales a manera de marca y en términos de performatividad, con espacios restringidos para la conformación política.

Dicho esto, regresamos a la pregunta inicial de esta investigación, sobre si podemos o no seguir hablando de una misión ciudadana de la universidad privada del siglo XXI. En la UEP, los discursos con respecto a la realidad social y al rol de la universidad en ella están bastante presentes, a pesar de la celebración de la individualización. Este compromiso se ve encarnado en la figura de los líderes y de los cursos de aprendizaje-servicio, en cuya formación la universidad está poniendo especial énfasis en los últimos años. Sin embargo, la relación entre universidad y sociedad no es la misma que la existente en medio de la politización de décadas anteriores. Estos estudiantes y profesores líderes entienden su compromiso con el cambio como la toma de acciones concretas, directas y con impactos medibles de mejora en la vida de las personas beneficiarias. No se trata, por lo tanto, de un compromiso político de largo aliento con el cambio de las estructuras de dominación ni tampoco con la crítica. El énfasis en la 
participación y la acción apabulla a los nuevos ciudadanos emprendedores, a quienes no se les brindan las herramientas ni los espacios para la discusión ni la reflexión. Ni las exclusiones que estas acciones puedan reproducir ni el cuestionamiento de las estructuras de poder que las legitiman forman parte de este repertorio, lo cual resulta preocupante sobre todo al tratarse de instituciones a las que se les encomienda la innovación y la reflexividad de nuestro proyecto como nación.

\section{Agradecimientos}

Este artículo está basado en la tesis de licenciatura que realicé para graduarme como antropóloga en la Pontificia Universidad Católica del Perú. Esta investigación no hubiera visto la luz sin la paciencia y acompañamiento de Gisela Cánepa, quien supo ver el potencial en mi propuesta, me ayudó a formularla y a encontrar las mejores herramientas teóricas para sostenerla. Asimismo, gracias a todos los miembros del Taller de Cultura, Persona y Poder, en particular, a Giacomo Bassillio, Walter Maradiegue, Alonso Quinteros, Carmen Ilizarbe y Eloy Seclén; sin sus atinadas críticas y comentarios no hubiese podido cocinar y darle contundencia a mis conclusiones. Esta tesis se debe entender como producto de este proceso de discusión conjunta. Por último, gracias a Ludwig Huber por ser un excelente jefe, y obligarme a pensar, reformular y descartar.

\section{Nota biográfica}

Leonor Lamas es licenciada en Antropología por la Pontificia Universidad Católica (PUCP) del Perú y estudiante de la maestría en Historia en la misma universidad. Ha trabajado como asistente de investigación en el Instituto de Estudios Peruanos en temas relacionados a educación y clases medias, y se desempeña como jefa de prácticas del pregrado en Antropología de la PUCP. 


\section{Referencias}

Adams, N. y Valdivia, N. (1994). Los otros empresarios: Ética de migrantes y formación de empresas en Lima. Lima: Instituto de Estudios Peruanos.

Ansión, J. (1993). La escuela en tiempos de guerra. Una mirada a la educación desde la crisis y la violencia ( ${ }^{\mathrm{a}} \mathrm{ed}$.). Lima: Tarea.

Biehl, J., Good, B. y Kleinman, A. (2007). Introduction: Rethinking subjectivity. En J. Biehl, B. Good, y A. Kleinman (Eds.), Subjectivity: Etnographic investigations (1-17). Los Angeles: University of California Press.

Binkley, S. (2007). Governmentality and lifestyle studies. Sociology Compass 1(1), 111-126.

(2009). The work of neoliberal governmentality: Temporality and ethical substance in the tale of two dads. Foucault Studies, (6), 60-78.

Cánepa, G. (2012). Marca Perú: Repertorios culturales e imágenes de peruanidad (artículo no publicado). Lima.

. (2013). Gestión municipal como marca. Cuadernos: Arquitectura $y$ Ciudad (16), 41-84.

Cotler, J. (2005). A manera de conclusión. Clases, Estado y nación ( $3^{\mathrm{a}}$ ed.) (335340). Lima: Instituto de Estudios Peruanos.

Danzelot, J. (1991). Pleasure in work. En G. Burchell, C. Gordon y P Miller (Eds.), The Foucault Effect: Studies in governmentality (251-290). Chicago: The University of Chicago Press.

de Soto, H. (1987). Capítulo VII: Conclusión. El otro sendero (6 ${ }^{a}$ ed.) (283-314). Lima: Instituto Libertad y Democracia.

Degregori, C. (2012). La década de la antipolítica: Auge y caída de Alberto Fujimori y Vladimiro Montesinos. Lima: Instituto de Estudios Peruanos.

Doi, T. (1986). The anatomy of Self: The individual versus the society. New York, Londres: Kodansha.

Dore, R. (1976). The diploma disease. Berkeley: University of California Press.

Flores Galindo, A. (1988). Epílogo: Sueños y pesadillas. Buscando un Inca (409411). Lima: Editorial Horizonte.

Foucault, M. (1987). Hermenéutica del sujeto. Madrid: La Piqueta. Ibérica.

(1990). Tecnologías del yo y otros textos afines. Barcelona: Paidós

(2008). Nacimiento de la Biopolítica: Curso en College de France (1978-1979). Buenos Aires: Fondo de Cultura Económica.

(1999). La gubernamentalidad. Foucault, Estética, ética y hermenéutica, (1978) Barcelona: Editorial Paidós.

(1988). El sujeto y el poder. Revista Mexicana de Sociología, Jul.Sep., 50(3), 3-20. 
Giddens, A. (1995). Modernidad e identidad del yo; el yo y la sociedad en la época contemporánea. Barcelona: Península.

Goffman, E. (1981). La presentación de la persona en la vida cotidiana. Buenos Aires: Amorrortu Editores.

Golte, J. y León Gabriel, D. (2011). Polifacéticos: Jóvenes limeños del siglo XXI. Lima: Instituto de Estudios Peruanos.

Guber, R. (2004). El salvaje metropolitano. Buenos Aires: Editorial Paidós.

Hall, S. y Jefferson, T. (1976). Resistance through rituals: Youth subcultures in post-war Britan. Londres: Hutchinson.

Hamann, T. (2009). Neoliberalism, governmentality and ethics. Foucault Studies, (6), 37-59.

Huber, L y Lamas, L. (2015). Deconstruyendo el rombo: consideraciones sobre la nueva clase media en el Perú (Informe de investigación pendiente de publicación). Instituto de Estudios Peruanos, Lima.

Ilizarbe, C. (1999). Todavía no somos quienes queremos ser: Construcciones sociales del amor y la pareja en jóvenes de sectores medios de Lima. En A. Panfichi y M. Valcárcel (Eds.), Juventud, Sociedad y Cultura (71-505). Lima: Red para el Desarrollo de las Ciencias Sociales en el Perú.

Instituto Nacional de Estadística e Informática (2011). II Censo Nacional Universitario 2010. Sistema de consulta de datos. Recuperado de http:// censos.inei.gob.pe/cenaun/redatam_inei/

Kogan, L. (2010). El deseo del cuerpo: Mujeres y hombres en la Lima contemporánea. Lima: Fondo Editorial del Congreso del Perú.

Lavado, P, Martínez, J. J. y Yamada, G. (2014). ¿Una promesa incumplida? La calidad de la educación superior universitaria y el subempleo profesional en el Perú (Documento de Trabajo 2014-021). Banco Central de Reserva del Perú, Lima.

Martínez, J. (2011). Subjetividad, política y multitud: Tres referentes para abordar a la juventud. En G. Muñoz Gonzáles (Ed.), Jóvenes, culturas y poderes (189-221). Manizales: Cinde.

Mauss, M. (1973). Techniques of the body. Economy and Society 2(1), 70-88. London: Routledge.

McDonald, K. (1999). Introduction: Subjectivity and social experience. Struggles for subjectivity: Identity, action and youth experience (1-14). Cambridge: Cambridge University Press.

Mckezie, J. (2001). Perform or else: From discipline to performance. New York: Routledge.

Mead, M. (1985). Educación y cultura en Nueva Guinea: Estudio comparativo de la educación entre pueblos primitivos. Barcelona: Editorial Paidós.

150 I Peters, M. (2001). Education, enterprise culture, and the entrepeunirial self: A Foucauldian perspective. Journal of Educational Enquiry 2(2), 58-71. 
Rabinow, P. (2007). Anthropological obsservation and self-formation. En J. Biehl, B. Good y A. Kleinman (Eds.), Subjectivity: Etnographic investigations (98-119). Los Angeles: University of California Press.

Read, J. (2009). A genealogy of homo-economicus: Neoliberalism and the production of subjectivity. Foucault Studies (6), 25-36.

Schnitman, F. (1998). Nuevos paradigmas, cultura y subjetividad. Buenos Aires: Editorial Paidós.

Seclén, E. (2012). ¿A dónde van los que quieren salir adelante? El perfil de los estudiantes de las nuevas universidades privadas en Lima. El caso de la Universidad César Vallejo (Tesis inédita de maestría). Pontificia Universidad Católica del Perú, Lima.

Taylor, H. (2001). Insights into participation from critica management and labour process perspectives. En B. Cooke y U. Kothari (Eds.), Participation: The new tyranny? (123-138). New York: Zed Books.

Turner, V. (1987). The Antrhopology of Performance. Washington: Johns Hopkins University Press.

Uccelli, F., García, M. y Montero, C. (2012) Rutas y barreras de jóvenes limeños: desigualdades y movilidades de los «incluidos» (Informe de investigación no publicado). Instituto de Estudios Peuanos, Lima.

Ulrich, B. y Beck-Gernsheim, E. (2002). Individualization: Institutionalized individualism and its social and political consequences. Londres: Sage.

Weber, M. (2001). La ética protestante y el espíritu del capitalismo. Madrid: Alianza Editorial. 